\title{
Pairwise comparison based ranking and scoring algorithms
}

Technical Report No. 223b

Ulf A. Hamster

orcid: 0000-0002-0440-4868

April 1, 2021

\begin{abstract}
The task is to convert a large sparse matrix of unbalanced paired comparison vote counts to scores for each item. Five procedures are presented and benchmarked. It is recommended to use the BTL algorithm for full batch computations (e.g. one-time quantitative studies), and the proposed p-value based procedure when scores must be partially updated in production deployments.
\end{abstract}

\section{Nomenclature}

\begin{tabular}{ccl}
\hline symbol & type & description \\
\hline \hline$C_{i}$ & - & Name of the $i$-th item (or resp. $i$-th choice) \\
$i, j$ & $\mathbb{N}_{>0}$ & Position index of the items $C_{i}$ and $C_{j}$ \\
$N_{i j}$ & $\mathbb{N}_{\geq 0}$ & Num. of times (count, frequency) users choose \\
& $C_{i}>C_{j}$ \\
\hline$\mu_{i j}$ & $\{x \in \mathbb{R} \mid 1 \geq x \geq 0\}$ & Simple ratio $\mu_{i j}=\frac{N_{i j}}{N_{i j}+N_{j i}}$ \\
$m_{i}$ & $\mathbb{R}$ & A metric associated with the $i$-th item \\
$s_{i}$ & $\{x \in \mathbb{R} \mid 1 \geq x \geq 0\}$ & Adjusted, scaled, calibrated scores \\
\hline$p_{i j}$ & $\{x \in \mathbb{R} \mid 1 \geq x \geq 0\}$ & Probability of error (p-value) of a Pearson's $\chi^{2}-$ \\
& & test \\
$q_{i j}$ & $\{x \in \mathbb{R} \mid 1 \geq x \geq 0\}$ & 1 - $p_{i j}$ if $N_{i j}>N_{j i}$ else zero \\
\hline$A$ & $\mathbb{R} \mathbb{R}^{d \times d}$ & Reciprocal pairwise comparison matrix $A=\left(a_{i j}\right)$ \\
$m$ & $\mathbb{R}$ & Eigenvalue \\
$x$ & $\mathbb{R}^{d}$ & Eigenvector $x=\left[x_{1}, x_{2}, \ldots, x_{d}\right]$ \\
\hline$G$ & $\mathbb{R} d \times d$ & The sparse generator matrix $G=\left(g_{k j}\right)$ \\
$\operatorname{Pr}(k \mid j)$ & $\{x \in \mathbb{R} \mid 1 \geq x \geq 0\}$ & Transition probability matrix \\
$\pi_{j}$ & $\{x \in \mathbb{R} \mid 1 \geq x \geq 0\}$ & Item (state) probability \\
$\pi_{k}$ & $\{x \in \mathbb{R} \mid 1 \geq x \geq 0\}$ & Predicted probability of $C_{k}>C_{j}$ \\
\hline $\operatorname{Pr}\left(C_{i}>C_{j}\right)$ & $\{x \in \mathbb{R} \mid 1 \geq x \geq 0\}$ & Probability of $C_{i}>C_{j}$ \\
$\gamma_{i}$ & $\{x \in \mathbb{R} \mid 1 \geq x \geq 0\}$ & Score to be estimated as BTL model parameter \\
\hline
\end{tabular}

Table 1: Used symbols and notations used in this report.

\section{Introduction}

This reports explores procedures to rank and score items based on pairwise comparison frequencies. The paired comparison matrix is expected to be extremely sparse (i.e. no 
exhaustive enumeration of all possible pairs), exhibit unbalanced frequencies or resp. unequal sample sizes (e.g. few pairs are often evaluated), and the number of items is much higher than the number of annotators (i.e. millions of items versus a couple of dozens of users). In other words, the algorithm must quickly process a large sparse matrix.

Computing sum of simple ratios is fast and scores might be good enough (Sec. 2). The p-value based metric to rank accommodates for unequal frequencies across different pairs (Sec. 3). Another idea is to estimate and simulate a transition matrix of the next item to be better (Sec. 5). For both the p-value and transition matrix based approach, we could not find literature. On the other side, using eigenvectors as scores (Sec. 4) and the Bradley-Terry-Luce (BTL) probability model (Sec. 6) are established algorithms to derive ranks and scores from paired comparisons.

\section{Sum of Simple Ratios}

The scoring procedure 1 has the following assumptions. First, the simple ratio $\mu_{i j}$ ignores the sample sizes $N_{i j}+N_{j i}$ across different pairs $(i, j)$. Thus, all observed pairs of the $i$-th item are weighted equally. Second non-observed pairs $\left(i^{*}, j^{*}\right)$ are assumed to be $\mu_{i^{*} j^{*}}=0$. For example diagonals are always $\mu_{i i}=0$. Thus, the score $s_{i}$ might be higher because more distinct pairs have been evaluated for the $i$-th item, i.e. explorative sampling might yield higher scores than exploitive sampling.

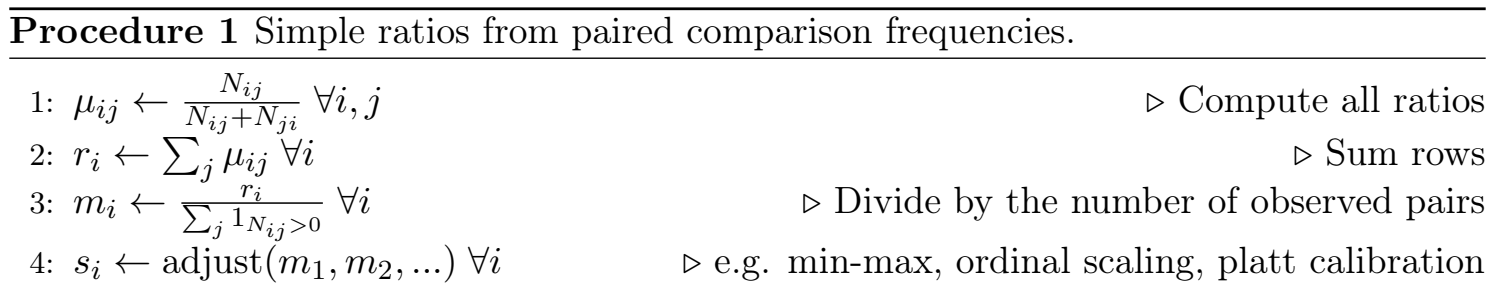

\section{3 p-value based Metric to Rank}

The question which opposing frequency $N_{i j}$ or $N_{j i}$ is larger, can be treated as hypothesis test (Eq. 1).

$$
\mu_{i j}=\frac{N_{i j}}{N_{i j}+N_{j i}} \quad, \quad H_{0}: \mu_{i j}=0.5 \quad, \quad H_{a}: \mu_{i j}>0.5
$$

Instead of the discrete binomial test, the Pearson's $\chi^{2}$-test should be used to compute the probability of error $p_{i j}$ (p-value). If $\mu_{i j}>0.5$ or resp. $N_{i j}>N_{j i}$, the smaller the p-value $p_{i j} \rightarrow 0$, the more significant the rejection of the null hypothesis $H_{0}$. In other words, pairs with small p-value should yield higher scores, i.e. $q=1-p$.

$$
q_{i j}=\left\{\begin{array}{lr}
1-p_{i j}, & \text { if } N_{i j}>N_{j i} \\
0, & \text { otherwise }
\end{array} \quad \forall i, j\right.
$$

The Eq. 2 has the advantage that a) non-observed pairs, and b) at least half of the elements can be stored in a sparse matrix format. Similar to procedure 1, we can compute the sum of each row $r_{i}=\sum_{j} q_{i j} \forall i$, divide it by the number of observed pairs, and adjust these values to the scores.

The Hoaglin Approximation [Hoa77] can be used to compute the p-values [Beh18, Eq. 6] 
what would simplify for zero degree of freedom to Eq. 4.

$$
\begin{aligned}
X^{2} & =\frac{\left(N_{i j}-E\right)^{2}}{E} \quad \text { with } E=\left(N_{i j}+N_{j i}\right) / 2 \\
p & \approx(0.1)^{\frac{\sqrt{X^{2}}+1.37266}{2.13161}}
\end{aligned}
$$

\section{Eigenvectors as scores}

Saa03 proposed to solve a pairwise comparison matrix as Eigenvalue-problem whereas the eigenvector can be interpreted as the items' scores.

1. Create a reciprocal pairwise comparison matrix $A=\left(a_{i j}\right)$ with

$$
a_{i j}=\left\{\begin{array}{ll}
N_{i j} / N_{j i}, & \text { if } N_{j i}>0 \\
0, & \text { otherwise }
\end{array} \quad \forall i, j\right.
$$

2. Solve the sparse Eigenvalue-problem $A x=m x$ with $m$ the eigenvalue and $x=$ $\left[x_{1}, x_{2}, \ldots, x_{d}\right]$ the eigenvector.

The sparse Eigenvalue-problem can solved with the scipy implementation [Vir+20] of ARPACK LSY98].

\section{$5 \quad$ Estimate and simulate a transition matrix}

The idea is to estimate transition probability of the next item to be better. In a first step the generator matrix $G=\left(g_{i j}\right)$ is derived from the observed paired comparison frequencies $N_{i j}$ (Line 1-4 in procedure 2). The transition probability matrix $\operatorname{Pr}(i \mid j)$ of items being evaluated $C_{i}>C_{j}$ is the matrix exponential of the generator matrix $\operatorname{expm}\left(g_{i j}\right)$ what can be computed with scipy's implementation [Vir+20, AH09] (Line 5). In a final step, the state probabilities $\pi_{i}$ that $C_{i}>C_{j}$ are simulated (Line 6-7) and used as scores.

Procedure 2 Compute the generator matrix $G=\left(g_{i j}\right)$, the transition matrix $\operatorname{Pr}(i \mid j)$, and simulate the probability that $\pi_{i}$.

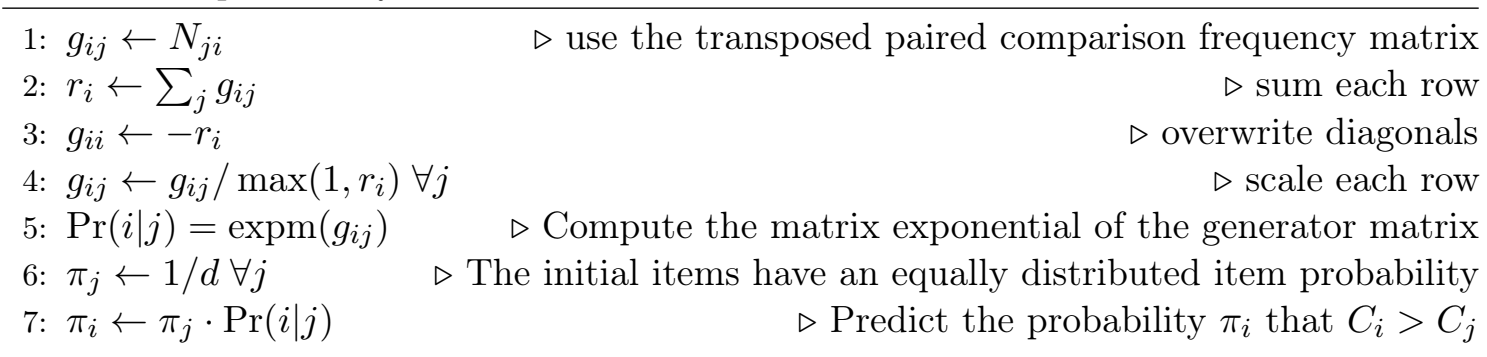

\section{Bradley-Terry-Luce (BTL) probability model}

Given $d$ the number of items, and each item has a parameters $\gamma_{i} \in\{x \in \mathbb{R} \mid 1 \geq x \geq 0\}$, the Bradley-Terry-Luce probability model $[\mathrm{BT} 52]$ is

$$
\operatorname{Pr}\left(C_{i}>C_{j}\right)=\frac{\gamma_{i}}{\gamma_{i}+\gamma_{j}}
$$


According to [Hun04, pp. 386-387] we can maximize the log-likelihood

$$
\max _{\gamma_{1}, \ldots, \gamma_{d}} \sum_{i=1}^{d} \sum_{j=1}^{d} N_{i j}\left[\ln \left(\gamma_{i}\right)-\ln \left(\gamma_{i}+\gamma_{j}\right)\right]
$$

by updating in $h=1,2, \ldots, H$ iteration steps

$$
\gamma_{i}^{(h+1)}=\operatorname{norm}\left[\sum_{j=1}^{d} N_{i j} \cdot\left(\sum_{j \neq i} \frac{N_{i j}+N_{j i}}{\gamma_{i}^{(h)}+\gamma_{j}^{(h)}}\right)^{-1}\right]
$$

with

$$
\operatorname{norm}\left(x_{1}, \ldots, x_{d}\right)=\frac{x_{i}}{\sum_{j=1}^{d} x_{j}}
$$

to ensure $\sum_{i=1}^{d} \gamma_{i}^{(h+1)}=1$.

\section{Experiment}

We extracted a paired comparison matrix from the sentiment dataset (see "BWS annotations") in [KM17] by using the PyPi package bwsample (v0.6.1) |Ham21]. The five different scores were compared with the reported scores in [KM17] (see "Scores obtained by BWS") that were computed according to [Orm09]. The Spearman rank correlations in Tab. 2 indicate that the Eigenvector procedure might have troubles what can be confirmed by manual inspection. The eigenvector values are very tiny what leads to numerical instabilities. To our surprise the sparse matrix based implementation of the BTL model with the MM algorithm is very fast.

\begin{tabular}{l|ccccc|c|}
\hline & $(\mathrm{b})$ & $(\mathrm{c})$ & $(\mathrm{d})$ & $(\mathrm{e})$ & (f) & time \\
\hline \hline (a) simple ratio & .9997 & -0.0133 & 1. & 1. & 1. & 1.000 \\
(b) approx p-value & - & -0.0110 & 1. & 1. & 1. & 0.730 \\
(c) eigenvector & - & - & 1. & 1. & 1. & 0.733 \\
(d) transitions & - & - & - & 1. & 1. & 12.077 \\
(e) BTL model & - & - & - & - & 1. & 0.743 \\
\hline (f) scores from KM17 & - & - & - & - & - & - \\
\hline
\end{tabular}

Table 2: Spearman rank correlations between the five procedures and the reference scores. The computation run time is represented as a multiple of the run time for computing the simple ratios based scores.

\section{Conclusion}

Among the transition matrix procedure (Sec. 5), eigenvector procedure (Sec. 4) and BTL model (Sec. 6), the latter should be used because it delivers numerical stable results, is fast to compute, and theoretical sound. However, all three procedures would require a complete re-computation if the frequency of just one pair was updated, i.e. an update is still $\mathcal{O}\left(n^{2}\right)$ complex. In case of simple ratios (Sec. 2) and p-value based metrics (Sec. 3) the intermediate results can be stored, e.g. the $\left(q_{i j}\right)$ matrix, and only the pairs of $i$-th row and $j$-the columns must be re-computed and summed up, i.e. an update is only $\mathcal{O}(2 n)$ complex. Therefore, it depends on the use case which procedure should be used. For a quantitative study, use the BTL model to compute the scores in on go. And deploy the p-value based metric as online algorithm in an production deployment. 


\section{References}

[BT52] Ralph Allan Bradley and Milton E. Terry. "Rank Analysis of Incomplete Block Designs: I. The Method of Paired Comparisons". In: Biometrika 39.3/4 (1952). Publisher: [Oxford University Press, Biometrika Trust], pp. 324-345. Issn: 00063444. DOI: 10.2307/2334029. (Visited on 03/25/2021).

[Hoa77] David C. Hoaglin. "Direct Approximations for Chi-Squared Percentage Points". In: Journal of the American Statistical Association 72.359 (1977), pp. 508-515. DOI: 10.2307/2286208. (Visited on 04/01/2021).

[LSY98] R. B. Lehoucq, D. C. Sorensen, and C. Yang. ARPACK Users' Guide. Software, Environments and Tools. Society for Industrial and Applied Mathematics, Jan. 1998. ISBN: 978-0-89871-407-4. DOI: 10.1137/1.9780898719628, URL: https: //epubs.siam.org/doi/abs/10.1137/1.9780898719628.

[Saa03] Thomas L. Saaty. "Decision-making with the AHP: Why is the principal eigenvector necessary". In: European Journal of Operational Research 145.1 (Feb. 2003), pp. 85-91. ISSN: 03772217. DOI: 10.1016/S0377-2217(02)00227-8.

[Hun04] David R. Hunter. "MM algorithms for generalized Bradley-Terry models". In: The Annals of Statistics 32.1 (Feb. 2004). Publisher: Institute of Mathematical Statistics, pp. 384-406. ISSN: 0090-5364, 2168-8966. DOI: 10.1214/aos/1079120141.

[AH09] Awad H. Al-Mohy and Nicholas J. Higham. "A New Scaling and Squaring Algorithm for the Matrix Exponential". In: SIAM Journal on Matrix Analysis and Applications 31.3 (Aug. 2009). Publisher: Society for Industrial and Applied Mathematics, pp. 970-989. ISSN: 0895-4798. DOI: 10.1137/09074721X. URL: https://epubs.siam.org/doi/10.1137/09074721X.

[Orm09] Bryan Orme. "MaxDiff Analysis: Simple Counting, Individual-Level Logit, and HB". en. 2009. URL: https://sawtoothsoftware.com/uploads/sawtoothsoftware/ originals/f89a6537-1cae-4fb5-afad-9d325c2a3143.pdf.

[KM17] Svetlana Kiritchenko and Saif Mohammad. "Best-Worst Scaling More Reliable than Rating Scales: A Case Study on Sentiment Intensity Annotation". In: Proceedings of the 55th Annual Meeting of the Association for Computational Linguistics (Volume 2: Short Papers). Vancouver, Canada: Association for Computational Linguistics, July 2017, pp. 465-470. DOI: 10.18653/v1/P17-2074

[Beh18] Eric Beh. "Exploring How to Simply Approximate the P-value of a Chi-squared Statistic". In: Austrian Journal of Statistics 47.3 (May 2018), pp. 63-75. ISSN: 1026-597X. DOI: 10.17713/ajs.v47i3.757. (Visited on 03/30/2021).

[Vir +20$]$ Pauli Virtanen et al. "SciPy 1.0: fundamental algorithms for scientific computing in Python". en. In: Nature Methods 17.3 (Mar. 2020). Number: 3 Publisher: Nature Publishing Group, pp. 261-272. ISSN: 1548-7105. DOI: 10.1038/s41592019-0686-2. URL: https://www . nature.com/articles/s41592-019-0686-2.

[Ham21] Ulf Hamster. bwsample: Sampling and Evaluation of Best-Worst Scaling sets. Version 0.6.1. Mar. 2021. DOI: 10.5281/zenodo.4603520. URL: https://doi org/10.5281/zenodo.4603520 